\title{
Cancer treatment-induced mucositis pain: strategies for assessment and management
}

\author{
Debra J Harris \\ Oregon Health Sciences University, \\ Portland OR, USA
}

\begin{abstract}
Mucositis pain is a major clinical problem associated with cancer treatment. Mucosal tissue injury is a dose-limiting side effect and also limits nutritional intake and oral function, resulting in weight loss and nutritional deficits for many patients. The pathophysiology of mucositis is thought to be a complex array of cytokine-mediated events, which begins with mucosal atrophy and eventually leads to the painful ulceration of the mucosa. This article reviews current research related to pain management for mucositis. Effective treatment for mucositis pain must be targeted at the various factors involved in the pain experience. Although a number of interventions aimed to prevent and treat mucositis have been studied, there is little evidence to recommend any one treatment modality. While current strategies for pain management rely on general treatment for acute pain, research developments are aimed at targeting the specific receptors and enzymes involved in mucositis. As these breakthroughs become available clinically, thorough assessment and timely directed interventions must be implemented in order to limit patient distress from mucositis. This article presents an assessment tool specific to mucositis pain, including physical, functional, and pain parameters.
\end{abstract}

Keywords: mucositis, pain management, stomatitis, assessment

\section{Introduction}

Chemotherapy agents continue to be the mainstay of cancer treatment, but side effects on highly proliferative tissues remain significant. Radiation therapy also affects rapidly dividing cells, putting patients receiving combined treatment modalities at increased risk for treatment-related toxicity. Among these is mucositis, a general term that describes the inflammatory response of mucosal epithelial cells to the cytotoxic effects of chemotherapy. Mucositis can affect all mucous membrane-covered surfaces from the mouth to the rectum (Camp-Sorrell 2000). Despite intestinal damage, much of the research and assessment tools continue to focus on oral sequela.

Mucositis can affect up to $100 \%$ of bone marrow transplant (BMT) patients, and is the most frequent serious side effect of therapy in the first 100 days after transplant (Epstein and Schubert 2004) and is the most common condition requiring system analgesics during cancer therapy (Epstein and Schubert 1999). Oral mucositis also affects up to $80 \%$ of patients receiving radiation for head and neck malignancies (Rubenstein et al 2004) and approximately 40\% of patients undergoing chemotherapy (Wojtaszek 2000). While the incidence and severity of mucositis may vary greatly among patient populations, it significantly reduces quality of life and patients' experience of treatment. Unresolved or untreated mucositis can lead to infections, impaired nutritional status, and other complications that can increase morbidity, and impact patient outcomes. Mucositis is a dose-limiting toxicity for both chemotherapy and radiation therapy, and therefore can directly impact survival (Avritsher et al 
2004). The need for opioid analgesics can cause extended hospital stays, which may in turn create further complications and increase costs.

The purpose of this paper is to explore pain mechanisms and treatments for pain associated with mucositis to better manage this difficult toxicity. A mucositis-specific pain assessment tool is also presented, with potential clinical interventions tailored to assessment findings.

\section{Mucositis and oral pain}

Pain, oral dysfunction, and gastrointestinal distress are commonly experienced by patients with mucositis, independent of the grade or severity. The rapid rate of mitosis in mucosal tissue makes it highly prone to injury from both chemotherapy and radiation. Mucositis can affect nutritional status, speech, comfort, and treatment compliance. The pain from oral mucositis has been reported as the most distressing symptom by patients receiving treatment for head and neck cancer, and chemotherapy (Rose-Ped et al 2002). Pain must be appropriately managed to best ensure positive outcomes for patients with mucositis.

Cancer treatment-induced mucositis causes acute pain, which is the result of sloughing of the epithelium, inflammation of the mucosa, and ulceration (Camp-Sorrell 2000). Tissue injury activates nociceptive receptors creating pain that resolves with the underlying tissue damage. The mechanism of pain perception and response are due to four distinct neural processes. Understanding the neurobiologic mechanisms of pain will allow for the development of therapeutic agents that target specific receptors, neurotransmitters and sites, which will improve management of mucositis pain.

BMT patients frequently describe acute oral pain as sore, burning, dull, and aching (McGuire et al 1998). In their pilot study, McGuire and colleagues (1998) use a framework based on the gate control theory of pain, introduced by Melzack and Wall (1965), which confirms the multidimensional nature of pain. Five dimensions are generally recognized, including physiologic, sensory, affective, cognitive, and behavioral dimensions. McGuire and colleagues (1998) added the sociocultural dimension as a sixth dimension of pain when evidence of the relationship between pain and its interference with daily activities, social relationships, and role performance was found. Because these pain dimensions exist for patients with mucositis, a better understanding of them can aid us in developing effective treatments.
Verbal descriptions of pain in the study by McGuire and colleagues (1998) most commonly included tender, sore, and irritating, with each word being mentioned almost 40 times over the study period. Patients' reports of overall pain relief during the study period suggested that their pain was only partially relieved. This finding suggests the need for careful and frequent nursing assessment and management. The authors conclude that multiple interventional approaches are needed to decrease the distress caused by acute oral pain and mucositis (McGuire et al 1998). A mucositis-specific pain protocol should address all elements of pain including tissue damage, sensitization of pain receptors, and elaboration of inflammatory and pain mediators (Epstein and Schubert 1999). These elements correspond to knowledge of the pathophysiology of mucosal tissue injury.

\section{Pathophysiology}

Recent developments in mucositis research indicate multiple factors which contribute to mucosal injury. The cytotoxic drugs most frequently associated with mucositis include bleomycin, cytarabine, doxorubicin, etoposide, 5-fluorouracil, ifosfamide, mercaptopurine, methotrexate, paclitaxel, vinblastine, vincristine, and vinorelbine (Dodd, Dibble, et al 2000; Raber-Durlacher et al 2000; Brown and Wingard 2004). High dose methotrexate, etoposide, and melphalan, as well as hyperfractionated radiation are implicated in the most severe forms of mucositis (Kwong 2004). Although these agents are all known to cause mucositis, there is still a great deal of patient variation in the severity of mucositis, even among patients receiving identical treatment regimens. Although it is not clear to what extent patient factors correlate with mucositis prevalence, several are identified in the literature, including age, nutritional status, and oral health. Other risk factors include: radiation treatment history; salivary gland dysfunction; physical, chemical and thermal mucosal injury; microbial flora; and graftversus host disease (Sonis 1998).

A number of biologic products impact the severity and extent of mucositis, including epidermal growth factor, tumor necrosis factor- $\alpha$, and cytokines such as granulocytemacrophage colony simulating factor and interleukin-1 (Epstein and Schubert 1999). Radiation and chemotherapy also alter normal oral microbial flora, and salivary quantity and composition (Turhal et al 2000). The combination of treatment-related effects and cytokines leads to mucosal 
atrophy, collagen breakdown, and eventual ulceration of the mucosa.

Sonis (2004) recently revised and expanded the phases of biologic development of mucositis. Whereas previously the clinical course of mucositis appeared to follow the same patter of suppression and recovery of white blood cells (Sonis 1998), recent work suggests that a complex array of biologic events generates mucosal tissue injury and healing. The events of the five phases are summarized in Table 1. These phases are interdependent and mediated by the action of cytokines, chemotherapy, oral bacterial flora, and bone marrow status. In the initial phase, free radicals break DNA strands and cause a small proportion of cells to die quickly. Tissue injury from activation of numerous biologic control mechanisms characterizes the second and third phases. Transcription factors such as nuclear factor- $\kappa \mathrm{B}$ are activated and upregulate genes that control synthesis of cytokines. Both chemotherapy and radiation treatment activate enzymes that increase the rate of apoptosis, which further adds to the chemical insults to the mucosa. Ulceration penetrating through the epithelium into the submucosa occurs in the fourth phase and creates the loss of function and pain typical of mucositis. Also in this phase, cell-wall products from colonizing bacteria stimulate macrophages to release additional cytokines. The fifth and final phase of mucositis is related to healing that occurs by migration, proliferation, and differentiation of epithelial cells (Sonis 2004). The complexity of this process contributes to the variety of patient experiences and the difficulties clinicians face in preventing and managing mucositis. As our understanding of these biologic events improves, more targeted therapies and interventions may be possible.

\section{Clinical manifestations}

The physical symptoms of mucositis begin 5 to 10 days after chemotherapy. According to Epstein and Schubert (1999), damage is often bilateral and involves nonkeratinized sites, including the buccal and labial mucosa, tongue, soft palate, and floor of the mouth. The gingival and hard palate are rarely involved (Camp-Sorrell 2000). Clinical manifestations progress from erythema, cracking, and inflammation, to pain, bleeding and ulceration. Pain may be present with any of these symptoms. The clinical presentation usually begins with mild focal changes, which can progress to marked tissue changes, bleeding, and pain (Raber-Durlacher et al 2000).

Epstein and Schubert note that during cancer treatment, oral mucositis is the most common condition that requires systemic analgesics. Intense mucositis pain can interfere with speaking, swallowing, and eating. As previously noted, additional sequele include bacterial and fungal infections, xerostomia, weight loss, dehydration, and nutritional deficiencies. Although oral complications are most frequently addressed in the literature, mucositis can occur along the entire gastrointestinal (GI) tract. According to McGuire (2002), there is almost no literature on pain associated with GI mucositis. Further research is necessary to establish the incidence, duration, and characteristics of pain related to different treatment regimens that cause mucosal tissue injury.

\section{Assessment}

To appropriately manage oral complications, specific, careful oral assessment is crucial for all patients, with special attention for patients at high risk of developing mucositis. Assessment of patients' oral health, physical, and nutritional status are important to identify risk factors and appropriate interventions. All of the assessment tools in the literature focus on oral assessment. There is very little mention, if at all, of symptoms in the esophagus or the gastrointestinal tract.

A number of assessment tools are available, many which have been developed for research purposes. These include the World Health Organization (WHO) grading scale, the oral assessment guide (OAG), the cancer and leukemia group B (CALGB) assessment guide and others listed in

Table I Biological stages of mucositis

\begin{tabular}{llll}
\hline Initiation & Message generation & Signaling and amplification & Ulceration \\
\hline $\begin{array}{lll}\text { Direct irreversible and } \\
\text { reversible damage to DNA }\end{array}$ & $\begin{array}{l}\text { Transcription factors such as } \\
\mathrm{NF}-\kappa \mathrm{B}, \text { upregulate a number } \\
\text { of genes in the epithelium, }\end{array}$ & Apoptosis and tissue injury & Epithelial integrity \\
& $\begin{array}{l}\text { Intact wound } \\
\text { causing increased production } \\
\text { of messaging and effector }\end{array}$ & Burface forms, \\
& proteins. & continued epithelial & sepsis possible. \\
& & &
\end{tabular}

Abbreviations: DNA, deoxyribonucleic acid; NF, nuclear factor. 
Table 2. These numerous assessment tools have a variety of strengths and weaknesses. The recently published clinical practice guideline for mucositis (Rubenstein et al 2004) notes that mucositis research does not use consistent scoring systems or consistent endpoints, which impedes progress aimed at prevention and treatment. Ideally an universally accepted assessment tool would be objective, demonstrate content validity and inter-user reliability. It also should be simple enough as to not be time-consuming for nurses or overwhelming to patients. The lack of consensus regarding accurate mucositis assessment is confounded when incorporating a thorough pain assessment. The current mucositis assessment tools do not incorporate pain assessment and this addition would be challenging. The range of assessment parameters (physiological, sensory, affective, cognitive, behavioral, and sociocultural) could become sufficiently cumbersome as to not be effective in clinical practice. For this reason, pain assessment tools widely used in clinical practice tend to focus on one dimension of pain. Visual analogue scales (VAS) or verbal descriptor scales (VDS) are written measures of pain intensity that are commonly used (Yeager 2000). The numeric (0 to 10$)$ verbal version of the VDS is also widely used and has been demonstrated to be reliable and valid. Yeager (2000) explains that considerations when choosing an appropriate pain assessment tool should include the relevant dimensions of pain, the purpose of the assessment (baseline vs ongoing), current pain interventions, and time and feasibility.

There is little information in the literature addressing assessment of mucositis-specific pain. The mucositis grading systems are partially based on the presence of pain, but do not address how this pain is assessed. These grading systems do not address severity, duration, or location of pain. An assessment tool developed specifically for mucositis pain which includes dimensions of pain, functional ability, and effectiveness of interventions is presented in Table 3. This tool has not yet been validated. However, it incorporates aspects of many validated tools. It allows documentation of pain locations and intensities on a 0-10 scale, and patients' ability to swallow, eat, and talk are scored as able, with difficulty, or unable. An area is provided for the nurse to document visual assessment and interventions. The patient's response to interventions is rated on a $0-4$ scale with 0 indicating a total response and 4 indicating significant worsening of symptoms. To use the tool effectively, nurses should implement it early in treatment. Thorough oral assessment should occur daily in the inpatient setting and at each outpatient appointment, with reassessment occurring during subsequent visits. This tool allows nurses in clinical practice to provide tailored interventions and assess the response to those interventions in a standardized way that can not occur with other available assessment tools.

\section{Management}

As with mucositis assessment, the wide variety of modalities aimed at prevention and treatment of mucosal injury creates a great deal of confusion for patients and clinicians alike. The section will summarize the most recent research findings, focusing on agents that have been studied using pain and functionality as endpoints.

\section{Basic oral care}

The purpose of basic oral care is to maintain the patient's baseline oral health and reduce the impact of cancer therapy on the oral mucosa. Good oral hygiene practices are thought to reduce pain, bleeding, infection, and dental complications (Rubenstein et al 2004). There is not a consensus on what constitutes a standard for oral care. The clinical practice guideline for mucositis (Rubenstein et al 2004) provides a recommendation level $\mathrm{B}$ that oral care protocols be implemented. Multiple studies (Graham et al 1993; Dibble et al 1996; Larson et al 1998; Yeager et al 2000) demonstrate

Table 2 Mouth assessment tools

\begin{tabular}{llll}
\hline Name of tool & Scale & Anatomic & Functional \\
measures & measures \\
\hline WHO Criteria & $0-4$ & Yes & Yes \\
OAG & $8-24$ & Yes & Yes \\
MacDibbs mouth assessment & $0-21$ & Yes & Yes \\
Spijkervet scoring system & $0-4$ & Yes & No \\
OMI & & Yes & Yes \\
CALGB assessment guide & $0-4$ & Yes & No \\
Lorentz mucositis index & $0-36$ & Yes & Schulz-Kindermann et al 2002 \\
\hline
\end{tabular}

Abbreviations: CALGB, cancer and leukemia group B; OAG, oral assessment guide; OMI, oral mucositis index; WHO, World Health Organisation. 
Table 3 Harris mucositis-related pain assessment tool

\begin{tabular}{llllll}
\hline Time & Pain & Swallow Eat Talk Visual assessment Intervention Response to intervention \\
\cline { 2 - 6 } & Location Intensity & & & \\
\hline
\end{tabular}

\begin{tabular}{|c|c|c|c|c|c|c|c|}
\hline Location & Intensity & Swallow & Eat & Talk & Visual assessment & Intervention & Response to intervention \\
\hline $\begin{array}{l}\text { Lips, } \\
\text { tongue, } \\
\text { mucosa, } \\
\text { gingiva, } \\
\text { esophagus, } \\
\text { stomach, } \\
\text { gut, anus }\end{array}$ & $0-10$ & $\begin{array}{l}\text { A-able } \\
\text { W-with } \\
\text { difficulty } \\
\text { U-unable }\end{array}$ & $\begin{array}{l}\text { A-able } \\
\text { W-with } \\
\text { difficulty } \\
\text { U-unable }\end{array}$ & $\begin{array}{l}\text { A-able } \\
\text { W-with } \\
\text { difficulty } \\
\text { U-unable }\end{array}$ & $\begin{array}{l}\text { Color, presence of ulcers, } \\
\text { red areas, white areas, } \\
\text { dryness } \\
\text { Middle-local }\end{array}$ & $\begin{array}{l}\text { Early-oral care, } \\
\text { saline or } \\
\text { bicarbonate rinses } \\
\text { I-some improveme } \\
\text { treatment such } \\
\text { as sucralfate or } \\
\text { benzydamine if } \\
\text { available } \\
\text { Late-systemic }\end{array}$ & $\begin{array}{l}\text { Patient rating based on decrease in } \\
\text { pain intensity, } \\
\text { 0-total response } \\
\text { 2-no difference } \\
\text { 3-some worsening of symptoms } \\
\text { 4-significant worsening of symptoms }\end{array}$ \\
\hline
\end{tabular}

the use of systematic protocols to improve patient outcomes. Components of an oral care protocol are outlined in Table 4.

\section{Topical agents}

Topical anesthetics such as viscous lidocaine are frequently combined with other agents to make mouthwashes. Other commonly used ingredients include dyphenhydramine, milk of magnesia, and chlorhexidine. To date, there is no significant evidence that these mouthwashes are effective (Rubenstein et al 2004). Dodd, Dibble, and colleagues (2000) performed a randomized clinical trial with 142 patients to evaluate the effectiveness of three different mouthwashes for chemotherapy-induced mucositis, and found evidence to support only routine oral hygiene, and the use of the inexpensive salt and soda mouthwash.

Turhal et al (2000) tested a mouthwash consisting of $125 \mathrm{ml}$ (100 mg) diphenhydramine, one ampule of $2 \%$ (100 mg) lidocaine, and two ampules of $8.4 \%$ sodium bicarbonate to $1000 \mathrm{ml}$ of sterile saline. This combination is easy to prepare and administer, and the ingredients are readily available at low cost. The subjects in the study $(n=31)$ were told to swish $20 \mathrm{~mL}$ of the solution inside their mouths and spit it out every 2 to 3 hours as needed. Scores were recorded for mucositis, bleeding, white blood cells, infection, taste, and metabolism according to the CALGB expanded common toxicity criteria. Findings support use of this mouthwash for relief of pain, however, the study sample is small and lacks a control group. The authors could only conclude that trials with other commercially available mouthwashes and new therapies are needed to determine the superiority of any particular regimen.

Other topical agents that have been studied related to pain management include sucralfate and dibucaine film. Sucralfate is an aluminum salt of sucrose orasulfate used to treat duodenal and gastric ulcer diseases. In addition to forming a protective physical barrier, sucralfate induces prostaglandin and mucus production, increases mucosal blood flow, and increases growth factor binding (Castagna et al 2001). Although it has been studied in ten randomized clinical studies, results are conflicting. Cengiz et al (1999) and Ertiz et al (2000) report reduction in mouth pain during feeding and a decrease in use of topical anesthetic or systemic analgesia respectively. Castagna et al (2001) reported a significant decrease in diarrhea in BMT patients treated with sucralfate and a higher caloric intake in this group. The current guideline (Rubenstein et al 2004) recommends that oral sucralfate not be used because of lack of evidence and potential for GI side effects. There is a level III grade $\mathrm{B}$ recommendation for sucralfate enemas to manage chronic radiation-induced proctitis with rectal bleeding, however. Further evaluation of the effectiveness of sucralfate is needed to determine its role in pain management and potential mucositis prophylaxis.

Phase III trials with benzydamine topical rinse show some promise both for pain relief and radiation-induced mucositis prevention. The current clinical practice guideline (Rubenstein et al 2004) includes a level I grade A recommendation for its use in patients receiving moderatedose radiation for head and neck cancer. Randomized trials 
Table 4 Oral care protocol

\begin{tabular}{|c|c|c|}
\hline Mouth care & Recommended intake & Avoid \\
\hline Floss once a day & Maintain appropriate fluid & Smoking \\
\hline Use a new, soft-bristled toothbrush once a month or with each chemo cycle & intake ( $1-3 \mathrm{~L} /$ day $)$ & - Rough, hard foods \\
\hline Brush for 90 seconds 3 times daily & Maintain nutritional status & Acidic foods \\
\hline Use fluoride toothpaste & Non-acidic fruits & (grapefruit, lemon \\
\hline \multirow[t]{2}{*}{ Rinse w/ bland mouth rinse, 30 seconds, before meals and before bedtime. Do not swallow. } & (banana, mango, melon, & orange) \\
\hline & peach) & · Alcohol \\
\hline \multirow[t]{3}{*}{ Keep lips lubricated } & & · Alcohol-containing \\
\hline & & and highly flavored \\
\hline & & oral care products \\
\hline
\end{tabular}

Note: Sources are: Larson et al 1998; Epstein and Schubert 1999; Rubenstein et al 2004.

indicate pain relief and reduced opioid use (Epstein and Schubert 2004). Other topical agents that may demonstrate a role in pain management include doxepin, a trycyclic antidepressant, topical morphine sulfate, and topical capsaicin. Further understanding of the mechanism of substance $P$ and inflammation will be necessary to determine the place these agents may have alongside established systemic analgesics.

\section{Growth factors}

Current understanding of the biologic process of mucosal injury reveals the potential role of a variety of growth factors for both prevention and treatment. A summary of growth factor mouthwash trials is included in Table 5 . The results of studies with agents such as tetrachlorodecaoxide, granulocyte-colony stimulating factor (GCSF), granulocytemacrophage colony-stimulating factor (GMCSF), and transforming growth factor-beta 3 indicate moderate benefits for pain relief and mucosal healing, however these agents are high-cost. Further research with expanded populations and standardized assessment tools and study endpoints is needed to identify the benefit of these agents.

A recent double-blind, placebo-controlled study of palifermin (recombinant human keratinocyte growth factor), which included 212 patients with hematologic cancers, indicates a number of positive outcomes for mucositis pain (Spielberger et al 2004). This growth factor mediates epithelial cell growth and repair and may decrease apoptosis, in addition to decreasing tumor necrosis factor- $\alpha$. Patients in the treatment arm of this study received palifermin 60 micrograms per kg per day IV for 3 days before and 3 days after transplant. A decrease in incidence of Grade 3 and Grade 4 mucositis ( $63 \%$ vs $98 \%$; $p<0.001$ ), and a decreased duration of mucositis (6 vs 9 days) were reported. A decrease in patient-reported soreness of the mouth and throat was also found, along with a decreased use of opioid analgesics (212 mg vs $535 \mathrm{mg}$ morphine equivalents). The use of total parenteral nutrition (TPN) was also less in the treatment group (Spielberger et al 2004).

\section{Systemic analgesics}

As described previously, mucositis is the most common side effect which requires systemic analgesia during cancer treatment. A survey of hospital pharmacists $(n=62)$, three institutions reported using intravenous opioids as initial therapy and four indicated opioids as second-line therapy (Mueller et al 1995). The current clinical practice guideline provides a Level I, Grade A recommendation for patientcontrolled analgesia (PCA) with morphine for patient undergoing hematopoietic stem cell transplant (HSCT). Although the guideline does not endorse PCA morphine for other patients with mucositis, it does include a recommendation to follow current guidelines for managing acute pain, such as the WHO analgesic ladder (Rubenstein et al 2004).

\section{Non-opioid strategies}

Current literature on pain includes the use of a variety of adjuvant treatments which can be used alone or in conjunction with opioids to improve the efficacy of pain management. Agents to consider include cycloogygenase-2 inhibitors, nonsteroidal antiinflammatory drugs, and gabapentin (Epstein and Schubert 2004). Cannabinoids, and alpha 2-adrenergic receptor agonists, including clonidine, nicotine, lidocaine, and ketamine may also be effective (Ripamonti and Dickerson 2001). The effectiveness of these agents specifically for pain relief needs further study.

Wong and Wilder-Smith (2002) evaluated the effects of low level laser therapy (LLLT) on the incidence and severity of chemotherapy-induced mucositis in patients $(n=15)$ who had developed Grade 3 or 4 mucositis during the previous chemotherapy cycle. Using a laser device emitting at $803 \mathrm{~nm}$, all intra-oral tissues were irradiated to provide total energy 
Table 5 Growth factor mouthwashes

\begin{tabular}{|c|c|c|c|c|}
\hline Reference & Growth factor & Type of study & Sample & Findings \\
\hline Malik et al 1997 & TCDO & $\begin{array}{l}\text { Double-blind, randomized, } \\
\text { placebo-controlled }\end{array}$ & $\begin{array}{l}62 \text { patients with mucositis, } \\
\text { all diagnoses }\end{array}$ & $\begin{array}{l}\text { Improved oral intake in } \\
\text { treatment group, shorter } \\
\text { subjective reports of pain }\end{array}$ \\
\hline Karthaus et al 1998 & G-CSF & Random, placebo-controlled & $\begin{array}{l}8 \text { patients over } 32 \text { chemo } \\
\text { cycles with high grade } \\
\text { lymphoma }\end{array}$ & $\begin{array}{l}\text { Significantly shorter hospital } \\
\text { stays, fewer episodes of } \\
\text { Grade } 4 \text { mucositis, earlier } \\
\text { recovery, reduction in need } \\
\text { for opioids in treatment } \\
\text { group }\end{array}$ \\
\hline Bez et al 1999 & GM-CSF & Pilot study & $\begin{array}{l}\text { I0 patients with mucositis, } \\
\text { BMT }\end{array}$ & $\begin{array}{l}\text { Treatment group more likely } \\
\text { to have symptoms lasting } \\
<9 \text { days }\end{array}$ \\
\hline Foncuberta et al 200I & TGF-beta 3 & $\begin{array}{l}\text { Phase II double-blind, } \\
\text { randomized, } \\
\text { placebo-controlled }\end{array}$ & $\begin{array}{l}\text { I } 25 \text { patients with solid } \\
\text { tumors or lymphomas }\end{array}$ & No effect \\
\hline Melichar et al 200I & GM-CSF & Pilot study & $\begin{array}{l}10 \text { patients with } \\
\text { chemotherapy-induced } \\
\text { mucositis, } 2 \text { I control } \\
\text { patients }\end{array}$ & $\begin{array}{l}\text { Oral administration improves } \\
\text { oral symptoms and } \\
\text { normalizes intestinal } \\
\text { permeability }\end{array}$ \\
\hline
\end{tabular}

Abbreviatons: BMT, bone marrow transplant; G-CSF, granulocyte colony-stimulating factor; GM-CSF, granulocyte-macrophage colony-stimulating factor; TCDO, chemically-stabilized chlorite-matrix;TGF, transforming growth factor.

densities of $0.8 \mathrm{~J} / \mathrm{cm}^{2}$. Laser treatment was preformed 24 hours before chemotherapy and once weekly until resolution of mucositis or until the next chemotherapy treatment cycle. In this study, 3 patients experienced Grade 1 to 2 mucositis, and one patient experienced Grade 3 to 4 mucositis, which interrupted treatment. While this modality shows positive results in this small, study, more research is needed to determine the effect of LLLT on pain and functionality as well as prevention of mucositis.

Other novel therapies are gaining interest for treatment and prevention of mucositis. Cryotherapy, or dissolving ice chips in the patient's mouth, has been recommended for patients undergoing bolus fluorouracil administration (Rubenstein et al 2004). Cryotherapy does not appear to be of benefit for other patients or for pain management. Lglutamine is an amino acid necessary for cell respiration in rapidly dividing cells. In a double-blind placebo trial $(\mathrm{n}=24)$ Anderson et al (1998) found that a $2 \mathrm{G} / \mathrm{m}^{2}$ twice daily swish and swallow formulation decreased the duration of mouth pain and the number of days of restricted oral intake. Preliminary phase III results indicate this agent may be effective for prevention (Peterson et al 2004). This agent also requires further study before treatment recommendations can be made. Gelclair ${ }^{\circledR}$ (OSI Pharmaceuticals, Melville, NY, USA) is a bioadherent gel consisting of sodium hyaluronate, polyvinylpyrrolidone, and glycyrrhetinic acid (Peterson et al 2004). Although results related to this agent are limited, it does appear to have a role in pain relief and is US Food and Drug Administration (FDA)-approved as a medical device.

\section{Key recommendations}

Mucositis is a common side effect with complex pathophysiology, assessment, and treatment strategies. Treatment is also confused by the use of "magic" mouthwashes and lack of uniform oral protocols. As research in this area continues to expand, systematic assessment including visual assessment, pain, and patient function is critical. Although publication of the first clinical practice guideline for mucositis assists in clarifying prevention and treatment methods, we must continue to provide patientappropriate interventions. Despite advances in research, no one agent has shown to be more efficacious than following current guidelines for acute pain management. Until novel therapies are shown to be effective, multiple, evidencedbased, interventional approaches are needed to decrease the emotional and physical distress caused by acute oral pain and mucositis. A mucositis-specific pain protocol should be developed to address all elements of pain including tissue damage, sensitization of pain receptors, and elaboration of inflammatory and pain mediators. Clinicians must continue to develop their understanding of the impact of the pain and distress mucositis has for patients. Mucositis management will improve through clinician and patient education, appropriate assessment, and adequate interventions. 


\section{References}

Anderson PM, Schroeder G, Skubitz KM.1998. Oral glutamine reduces the duration and severity of stomatitis after cytotoxic cancer chemotherapy. Cancer, 74:2879-84.

Avritscher EB, Cooksley CD, Elting LS. 2004. Scope and epidemiology of cancer therapy-induced oral and gastrointestinal mucositis. Semin Oncol Nurs, 20:3-10.

Bez C, Demarosi F, Sardella A, et al. 1999. GM-CSF mouthrinses in the treatment of severe oral mucositis. Oral Surg Oral Med Oral Pathol Oral Radiol Endod, 88:311-15.

Brown CG, Wingard J. 2004. Clinical consequences of oral mucositis. Semin Oncol Nurs, 20:16-21.

Camp-Sorrell D. 2000. Chemotherapy: toxicity management. In: Yarbro $\mathrm{CH}$, Frogge MH, Goodman M, et al. (eds). Cancer nursing principles and practice. 5th ed. Boston: Jones \& Bartlett. p 444-86.

Castagna L, Benhamou E, Pedraza E, et al 2001. Prevention of mucositis in bone marrow transplantation: A double blind randomized controlled trial of sucralfate. Ann Oncol, 12:953-55.

Cengiz M, Ozyar E, Ozturk D, et al. 1999. Sucralfate in the prevention of radiation-induced oral mucositis. J Clin Gastroenterol, 28:40-3.

Dibble SL, Shiba G, MacPhail L, et al. 1996. MacDibbs mouth assessment: a new tool to evaluate mucositis in the radiation therapy patient. Cancer Pract, 4:135-40.

Dodd MJ, Dibble SL, Miaskowski C, et al. 2000. Randomized clinical trial of the effectiveness of 3 commonly used mouthwashes to treat chemotherapy-induced mucositis. Oral Surg Oral Med Oral Pathol Oral Radiol Endod, 90:39-47.

Epstein JB, Schubert MM. 1999. Oral mucositis in myelosuppressive cancer therapy. Oral Surg Oral Med Oral Pathol Oral Radiol Endod, 88:273-6.

Epstein JB, Schubert MM. 2004. Managing pain in mucositis. Semin Oncol Nurs, 20:30-7.

Ertiz D, Erkal HS, Serin M, et al. 2000. Clinical and histopathological evaluation of sucralfate in prevention of oral mucositis induced by radiation therapy in patients with head and neck malignancies. Oral Oncol, 36:116-20.

Foncuberta MC, Cagnoni PJ, Brandts CH, et al. 2001. Topical transforming growth factor-beta 3 in the prevention or alleviation of chemotherapyinduced oral mucositis in patients with lymphomas or solid tumors. $J$ Immunother, 24:384-8.

Graham K, Pecoraro D, Ventura M. 1993. Reducing the incidence of stomatitis using a quality assessment and improvement approach. Cancer Nurs, 16:117-22.

Harris DJ, Knopf MT. 2004. Assessing and managing chemotherapyinduced mucositis pain. Clin J Oncol Nurs, 8:622-8.

Karthaus M, Rosenthal C, Huebner G, et al. 1998. Effect of topical oral G-CSF on oral mucositis: A randomised placebo-controlled trial. Bone Marrow Transplant, 22:781-5.

Kwong KF. 2004. Prevention and treatment of oropharyngeal mucositis following cancer therapy. Cancer Nurs, 27:183-205.

Larson PJ, Miaskowski C, MacPhail L, et al. 1998. The PRO-SELF Mouth Aware program: An effective approach for reducing chemotherapyinduced mucositis. Cancer Nurs, 21:263-8.
Malik IA, Moid I, Haq S, et al. 1997. A double-blind, placebo-controlled, randomized trial to evaluate the role of tetrachlorodecaoxide in the management of chemotherapy-induced oral mucositis. J Pain Symptom Manage, 14:82-7.

Melzack R, Wall PD. 1965. Pain mechanisms: a new theory. Science, 150:971-9.

McGuire D. 2002. Mucosal tissue injury in cancer therapy. Cancer Pract, 10:179-90.

McGuire DB, Yeager KA, Dudley WN, et al. 1998. Acute oral pain and mucositis in bone marrow transplant and leukemia patients: Data from a pilot study. Cancer Nurs, 21:385-93.

Melichar B, Kohout P, Bratova M, et al. 2001. Intestinal permeability in patients with chemotherapy-induced stomatitis. J Cancer Res Clin Oncol, 127:314-18.

Mueller BA, Millheim ET, Farrington EA, et al. 1995. Mucositis management practices for hospitalized patients: National survey results. J Pain Symptom Manage, 10:510-20.

Peterson DE, Beck SL, Keefe DM. 2004. Novel therapies. Semin Oncol Nurs, 20:53-8.

Raber-Durlacher JE, Weijl NI, Abu Saris M, et al. 2000. Oral mucositis in patients treated with chemotherapy for solid tumors: A retrospective analysis of 150 cases. Support Care Cancer, 8:36671.

Ripamonti C, Dickerson ED. 2001. Strategies for the treatment of cancer pain in the new millennium. Drugs, 61:955-77.

Rose-Ped AM, Bellm LA, Epstein JB, et al. 2002. Complications of radiation therapy for head and neck cancers: The patient's perspective. Cancer Nurs, 25:468-9.

Rubenstein EB, Peterson DE, Schubert M, et al. 2004. Clinical practice guidelines for the prevention and treatment of cancer therapy-induced oral and gastrointestinal mucositis. Cancer, 100:2026-46.

Schulz-Kindermann F, Hennings D, Ramm G, et al. 2002. The role of biomedical and psychosocial factors for the prediction of pain and distress in patients undergoing high-dose therapy and BMT/PBSCT. Bone Marrow Transplant, 29:341-51.

Sonis ST. 1998. Mucositis as a biological process: A new hypothesis for the development of chemotherapy-induced stomatotoxicity. Oral Oncol, 34:39-43.

Sonis ST. 2004. A biological approach to mucositis. J Support Oncol, 2:21-32.

Spielberger R, Stiff P, Bensinger W, et al. 2004. Palifermin for oral mucositis after intensive therapy for hematologic cancers. $N$ Eng $J$ Med, 351:2590-8.

Turhal NS, Erdal S, Karacay S. 2000. Efficacy of treatment to relieve mucositis-induced discomfort. Support Care Cancer, 8:55-8.

Wojtaszek C. 2000. Management of chemotherapy-induced stomatitis. Clin J Oncol Nurs, 4:263-70.

Wong SF, Wilder-Smith P. 2002. Pilot study of laser effects on oral mucositis in patients receiving chemotherapy. Cancer $J$, 8:24754.

Yeager KA, McGuire DB, Sheidler YR. 2000. Assessment of cancer pain. In: Yarbro CH, Frogge MH, Goodman M, et al. (eds). Cancer nursing principles and practice. 5th ed. Boston: Jones \& Bartlett. p 633-56. 